\title{
Freestyle root replacement for complex destructive aortic valve endocarditis
}

\author{
Anneliese Heinz, MD, Julia Dumfarth, MD, Elfriede Ruttmann-Ulmer, MD, Michael Grimm, MD, and
} Ludwig C. Müller, MD

Objectives: In destructive aortic valve endocarditis with abscess formation in the root, homografts are used more often than xenografts. Because we had reliable perioperative results with Freestyle (Medtronic Inc, Minneapolis, Minn) xenograft root replacement in these complex patients, we analyzed the long-term outcome in this high-risk indication.

Methods: Of 126 consecutive patients with aortic valve endocarditis treated by surgery from 1997 to 2012,32 $(25.4 \%)$ received a Freestyle aortic root replacement for severe, destructive valve endocarditis and were studied retrospectively with approval of the local ethical committee. Perioperative complications, recurrence of endocarditis, and long-term morbidity and mortality were analyzed. The follow-up period was 3 months to 11.5 years.

Results: Indication for surgery was native $(\mathrm{n}=9)$ and prosthetic valve endocarditis $(\mathrm{n}=23)$. In 18 patients, concomitant procedures were performed: coronary bypass $(n=9)$, additional valve surgery $(n=6)$, and ascending aortic surgery $(n=7)$. Thirty-day mortality was $19.4 \%(n=6)$. There were no instances of technical failure requiring modification of the surgical strategy or reoperation for anastomotic bleeding. Actuarial survival at 5 and 10 years was $61.9 \%$ and $54.2 \%$, respectively. Freedom from death, reoperation for prostheses dysfunction, and recurrence of endocarditis as the composite end point at 5 and 10 years was $56.3 \%$ and $53.1 \%$, respectively.

Conclusions: The Freestyle root was used successfully with no technical complications in all patients with most severe destructive aortic root endocarditis. In view of this complex patient population, short- and long-term results make this conduit a reliable choice for treatment of this condition. (J Thorac Cardiovasc Surg 2014;147:1265-70)

Surgery for severe infective endocarditis (IE) is still challenging and associated with significant morbidity and mortality. ${ }^{1}$ The choice of the optimum conduit for aortic root replacement in destructive endocarditis with annular, supra-annular, or infra-annular abscess formation depends on technical considerations, graft resistance to infection, and durability of the graft. Homografts, pericardial patch repair with prosthetic valve replacement, prosthetic valved conduits, and xenografts may be used.

According to American College of Cardiology/American Heart Association guidelines, an aortic homograft is considered the gold standard, and especially aortic valve re-replacement in patients with active prosthetic valve endocarditis (PVE) is recommended to be performed with a homograft (class of recommendation IIa, level of evidence C). ${ }^{1}$ In contrast, European Society of Cardiology guidelines advise valve replacement in aortic valve IE by a mechanical

\footnotetext{
From the Department of Heart Surgery, Medical University Innsbruck, Innsbruck, Austria.

Disclosures: Authors have nothing to disclose with regard to commercial support.

Received for publication Feb 15, 2013; revisions received April 14, 2013; accepted for publication May 2, 2013; available ahead of print July 15, 2013.

Address for reprints: Ludwig C. Müller, MD, Anichstrasse 35, A-6020 Innsbruck, Austria (E-mail: ludwig.mueller@i-med.ac.at). $0022-5223 / \$ 36.00$

Copyright (c) 2014 by The American Association for Thoracic Surgery http://dx.doi.org/10.1016/j.jtcvs.2013.05.014
}

or biological prosthesis. The use of cryopreserved or sterilized homografts is not recommended explicitly but might reduce the risk of persistent or recurrent infection. However, mechanical prostheses and xenografts compare favorably with the advantage of improved durability. Homografts or stentless xenografts are considered comparable and may be preferred in PVE or in cases where there is extensive aortic root destruction with aortoventricular discontinuity. ${ }^{2}$

Because of promising results in smaller series ${ }^{3-5}$ and the lack of long-term studies, we aimed to investigate perioperative complications caused by technical problems inherent to the graft, recurrence of endocarditis, and long-term outcome in patients who had a Freestyle (Medtronic Inc, Minneapolis, Minn) xenograft root implantation for severe excavating aortic valve endocarditis.

\section{MATERIALS AND METHODS Patients}

A total of 126 consecutive patients with a diagnosis of acute valve endocarditis were treated by surgery from 1997 to 2012 at our department Of these, 32 patients $(25.4 \%)$ with most severe destructive aortic root endocarditis had Freestyle xenograft full root replacement between 1997 and 2012 constitute the population of this study. The study was approved by the local ethical committee, and individual patients' informed consents were obtained.

IE was diagnosed according to modified Duke Criteria ${ }^{6}$ based on clinical signs, blood culture, histologic, examination, and transesophageal 


$$
\begin{aligned}
& \text { Abbreviations and Acronyms } \\
& \begin{aligned}
\mathrm{EF} & =\text { ejection fraction } \\
\mathrm{IE} & =\text { infective endocarditis } \\
\mathrm{LVOT} & =\text { left ventricular outflow tract } \\
\mathrm{NVE} & =\text { native valve endocarditis } \\
\mathrm{PVE} & =\text { prosthetic valve endocarditis }
\end{aligned}
\end{aligned}
$$

echocardiography. Indication for surgery in these patients was based on the transesophageal echocardiography results of root abscess or intracardiac fistula formation. Severe excavating aortic valve endocarditis was defined by intraoperative findings of acute necrotizing endocarditis with vegetations and partial or total destruction of the annulus and left ventricular outflow tract (LVOT).

Identification of the infective germ was done from cultures of blood or surgical specimens. Patients were treated with appropriate antibiotics before and after operation.

Native valve endocarditis (NVE) with abscess or fistula formation was present in 9 patients, and 23 patients underwent operation for PVE with annulus destruction. The previous procedure in these cases had been an aortic valve replacement with 13 biological prostheses, 9 mechanical valve prostheses, and 1 homograft valve. Additional procedures had been ascending aorta replacement $(\mathrm{n}=3)$, ascending aorta reduction plasty $(\mathrm{n}=2)$, coronary artery bypass grafting $(\mathrm{n}=4)$, and mitral valve repair or replacement $(\mathrm{n}=2)$. Nineteen patients had 1 previous aortic valve surgery, 3 patients had 2 previous aortic valve surgeries, and 1 patient had 4 previous aortic valve surgeries. The prior procedures had been performed 27 days to 36 years (median, 5.5 years) before Freestyle root replacement. Six patients had a history of cerebrovascular events that had occurred between 1 day and 8 years (median, 1.5 years) before; 4 events were a septic embolism related to IE.

Technical success of surgery (successful reconstruction of the aortic root and LVOT without need for early reoperation), perioperative complications (eg, mortality and early recurrence of endocarditis), and long-term outcome regarding survival, late PVE, relapse or reinfection, and late prosthesis dysfunction due to degeneration needing reoperation were analyzed.

Perioperative mortality was defined as mortality within 30 days or during hospital stay. Late mortality was defined as death thereafter. Follow-up period was 3 to 150 months (median, 20 months) and 100\% complete, including clinical and echocardiographic examinations. The standard schedule was to follow up the patients yearly at our cardiology department, including transthoracic echocardiography. Few patients were examined by their local physicians, and the results were sent to us. In any case, at least 1 recent follow-up ( $<3-6$ months) was required.

\section{Surgical Technique}

Mild hypothermic cardiopulmonary bypass $\left(32^{\circ} \mathrm{C}\right)$ was established using bicaval and ascending aortic cannulation. Myocardial protection was accomplished by retrograde (coronary sinus) and, if feasible, antegrade (by intubation of the coronary ostia) blood cardioplegia and topical cooling. Delivery of retrograde cardioplegia was repeated every 20 to 25 minutes. Complete transection of the aorta was performed after preliminary evaluation of the extent and severity of the disease, and radical resection of all infected and necrotic tissue was done without consideration of the extent of the created defect and later reconstruction or creation of heart block. Typically, the full extent of the destructive process became evident only after radical debridement. Specimens of the valve and necrotic material were sent for bacteriologic and histopathologic investigation. Reconstruction of the LVOT and aortic root was accomplished by means of a Freestyle xenograft aortic root. Because the aortic annulus was invariably destroyed by the inflammatory process, full root replacement with reimplantation of the coronary ostia using the button technique had to be performed. The graft was sutured to the LVOT with interrupted or running 4-0 Prolene sutures or a combination of both depending on the surgeon's preference and the individual situation. Interrupted sutures were usually tied over a strip of pericardium to prevent leakage. In 6 cases, a patch of pericardium had to be used to repair the LVOT at the level of the aorto-mitral curtain, and then the xenograft was sutured to the pericardium; 5-0 Prolene running sutures were used for implantation of the coronary buttons. For the distal anastomosis between the xenograft and the ascending aorta, a 4-0 Prolene running suture was used, which in all but the initial cases was buttressed by a strip of pericardium to prevent bleeding from the xenograft. Intracardiac defects also were closed with pericardium. Generally, $0.5 \%$ glutaraldehyde fixed autologous or bovine pericardium was used. The same material was used to repair defects of the mitral valve leaflets if present. Additional replacement of the ascending aorta with a homograft or Dacron aortic prosthesis was necessary in 3 patients.

\section{Statistical Analysis}

Continuous variables were expressed as median and range or mean \pm standard deviation, and categoric variables were expressed as percentages. Differences of categoric variables were calculated with the Fisher exact test, estimates of survival and incidence of valve-related complications were calculated using the Kaplan-Meier or log-rank method, and differences in continuous variables were calculated with the Student $t$ test. Statistical analysis was performed using SPSS version 18.0 (SPSS, Inc, Chicago, Ill).

\section{RESULTS}

Thirty-two patients received a Freestyle xenograft aortic root replacement with or without additional procedures; 22 patients were men $(68.8 \%)$, and 10 were women. The median age was 61 years (range, 16-79 years). Demographics and preoperative risk factors are shown in Table 1.

Mean bypass (cardiopulmonary bypass) time was $283 \pm$ 84 minutes, and mean crossclamping (aortic crossclamp) time was $172 \pm 40$ minutes. In 9 patients, hypothermic circulatory arrest was required for $5 \pm 11$ minutes. Replacement of the ascending aorta due to type A dissection was required in 1 patient, and the necessary re-replacement of the ascending aorta was performed with an open distal anastomosis with antegrade brain perfusion in 1 patient. In 7 patients, repeated short episodes of hypothermic circulatory arrest were required for control of bleeding during dissection or after release of the aortic clamp. Median intensive care unit stay was 5 days (range, 1-136 days), median ventilation time was 2 days (range, 1-115 days), and hospital stay was 26 days (range, 21-136 days).

The xenografts implanted in 32 patients ranged from 21 to $29 \mathrm{~mm}$ in size: $21 \mathrm{~mm}$ in 4 patients, $23 \mathrm{~mm}$ in 13 patients, $25 \mathrm{~mm}$ in 8 patients, $27 \mathrm{~mm}$ in 6 patients, and $29 \mathrm{~mm}$ in 1 patient. Eighteen patients $(56.3 \%)$ had concomitant surgical procedures: coronary artery bypass grafting $(n=9)$, mitral valve replacement or repair $(n=3)$, tricuspid valve repair $(\mathrm{n}=2)$, pulmonary valve replacement $(\mathrm{n}=1)$, LVOT patch repair $(\mathrm{n}=6)$, ascending aorta replacement $(\mathrm{n}=3)$, and ascending aorta reduction plasty $(\mathrm{n}=4)$ (Table 2). 
TABLE 1. Patient demographics

\begin{tabular}{lc}
\hline $\mathrm{n}$ & 32 \\
Age $(\mathrm{y}$, mean $\pm \mathrm{SD})$ & $58.5 \pm 15.3$ \\
Male sex (n) & 22 \\
Arterial hypertension (n) & 21 \\
Preoperative positive blood cultures (n) & 13 \\
EF $(\%$, mean \pm SD) & $49.1 \pm 11.6$ \\
Preoperative renal failure with hemofiltration/dialysis (n) & 6 \\
History of cerebrovascular accident (n) & 7 \\
Previous cardiac operation (n) & 23 \\
Active endocarditis (n) & 32 \\
Abscess/fistula (n) & 29 \\
\hline
\end{tabular}

$E F$, Ejection fraction; $S D$, standard deviation.

Microbiological testing was positive in 13 blood samples or cultures of the resected valves. No molecular biological investigations of specimens were performed. The most frequent germ was Staphylococcus aureus $(\mathrm{n}=7)$, followed by Staphylococcus epidermidis $(\mathrm{n}=1)$, Enterococcus faecalis $(\mathrm{n}=3)$, Aspergillus $(\mathrm{n}=1)$, and Klebsiella pneumonia $(\mathrm{n}=1)$. Blood cultures and valves remained sterile in 13 patients, and the result was unknown in 6 patients. Every patient was treated with antibiotics according to sensitivity testing or broad spectrum antibiotics, if therapy was empiric. A second- or third-generation cephalosporin, aminoglycoside, and in most cases metronidazole were used in most cases. The antibiotic therapy was started on the day of diagnosis and continued for 3 to 6 weeks after surgery consistent with recent European Society of Cardiology Guidelines (2) (NVE 2-6 weeks, PVE $>6$ weeks).

\section{Complications}

Perioperative mortality was $18.8 \%(\mathrm{n}=6)$ and not significantly different in patients undergoing operation for PVE $(5 / 23)$ or NVE $(1 / 9)(P=.648)$. Between postoperative days 1 and 5 , these patients died of multiorgan failure $(\mathrm{n}=2)$, cardiac decompensation $(\mathrm{n}=1)$, ischemic heart failure $(\mathrm{n}=1)$, or acute cardiac death $(\mathrm{n}=2)$. Although aortic crossclamp time was not different between survivors and nonsurvivors (mean $176 \pm 42$ vs $163 \pm 38$ minutes, $P=.806$ ), cardiopulmonary bypass time showed a significant difference between the 2 groups (mean $268 \pm 74$ vs $351 \pm 98$ minutes, $P=.027$ ).

Three patients $(9.4 \%)$ required reoperation for bleeding due to coagulopathy; 2 patients had 1 reoperation and 1 patient had 4 reoperations. However, no anastomotic bleeding at the site of the xenograft was detectable, and no complication related to the Freestyle prosthesis was found. Three patients required postoperative mechanical circulatory support $(9.3 \%, 2$ of them died), 2 with an intra-aortic balloon pump and 1 by extracorporeal membrane oxygenation; 3 patients $(9.3 \%)$ received a permanent pacemaker for new heart block; 6 patients $(18.8 \%)$ required temporary hemofiltration for new-onset acute renal failure; and 2 other
TABLE 2. Concomitant procedure

\begin{tabular}{lr}
$\mathrm{n}$ & 32 \\
Coronary artery bypass graft (n) & 9 \\
Mitral valve surgery (n) & 3 \\
Tricuspid valve surgery (n) & 2 \\
Pulmonary valve surgery (n) & 1 \\
LVOT patch repair (n) & 6 \\
Ascending aortic replacement (n) & 3 \\
Ascending aortic reduction plasty (n) & 4 \\
\hline LVOT, Left ventricular outflow tract. &
\end{tabular}

patients were already on preoperative long-term hemodialysis for chronic renal failure. There were no instances of early xenograft failure requiring reoperation and replacement of the xenograft in the postoperative period.

\section{Thromboembolic Events}

One patient had ischemic stroke caused by multiple embolic lesions of different age. These lesions were detected with a cerebral magnetic resonance tomography 14 days after surgery initiated by clinical symptoms. The patient presented with decreased motor function of the left arm and both legs. Follow-up after 3 months with a control magnetic resonance tomography showed declining cerebral findings. He gained full recovery of his motor function within 8 months with no gross neurologic residue.

\section{Long-Term Results}

Actuarial survival at 10 years was $54.2 \%$ (Figure 1) and did not differ for PVE (56.5\%) and NVE (55.5\%) $(P=.506)$. Total observed late mortality was $25 \%(\mathrm{n}=8)$. These patients died on days 94,106 , and 153 , and after 1.1, $3.4,6,10.5$, and 11 years as a result of acute myocardial infarction $(\mathrm{n}=1)$, unexplained sudden death $(\mathrm{n}=2)$, cardiac decompensation $(\mathrm{n}=2)$, brainstem infarction $(\mathrm{n}=1)$, Staphylococcus sepsis $(\mathrm{n}=1)$, and unknown reasons $(\mathrm{n}=1)$, respectively.

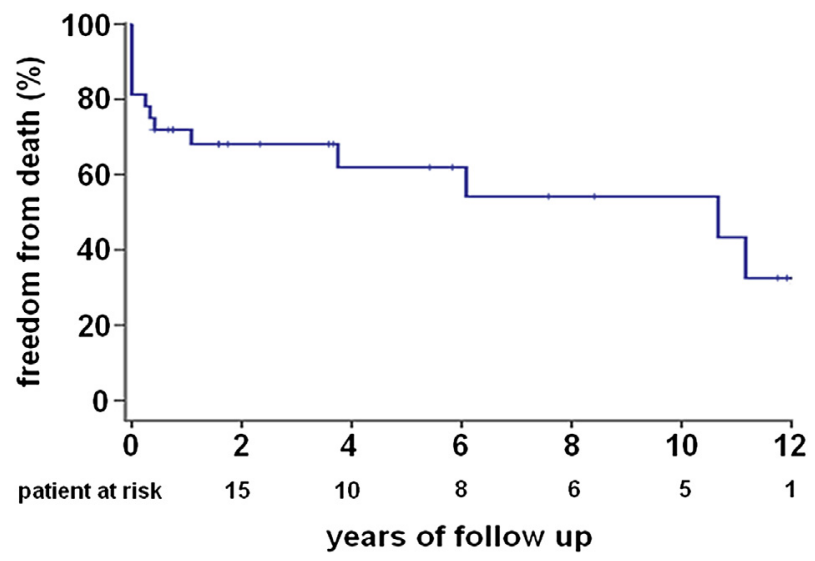

FIGURE 1. Actuarial survival after Freestyle root replacement for root endocarditis. 


\section{Valve-Related Events}

During follow-up, 3 patients underwent reoperation: 1 after 3 years and 1 after 12 years for xenograft degeneration $(\mathrm{n}=2,6.3 \%)$ and 1 after 3 years for recurrent endocarditis $(\mathrm{n}=1,3.1 \%)$. E faecalis had been cultivated at the initial procedure for PVE; at reoperation, blood cultures and the explanted xenograft remained sterile. The xenograft was replaced by a mechanical composite graft (Valsalva $21 \mathrm{~mm}$; St Jude Medical Inc, St Paul, Minn), and additional venous coronary bypass grafting was performed to the left anterior descending, obtuse marginal, and right coronary arteries. Because no germ could be cultivated, it remains unclear whether a relapse or re-IE occurred in this case. From the clinical, macroscopic, and histologic aspect, however, there was no doubt that IE was present. In the 2 other cases, the xenograft was degenerated with severe calcific stenosis and replaced by a mechanical conduit (St Jude Medical $21 \mathrm{~mm}$ [St Jude Medical Inc] and Carbo-Seal $21 \mathrm{~mm}$ [Sorin SpA, Milan, Italy]) by the Bentall procedure. The explanted xenografts remained sterile. One of the latter cases with PVE at the previous procedure but not at reoperation required additional pericardial patch repair of the LVOT and right pulmonary artery because of concretion of the Freestyle xenograft with the pulmonary artery. In the other patient, in whom the initial procedure was performed for NVE, a single coronary bypass graft to the right coronary artery was necessary.

Echocardiographic results (transthoracic echocardiography) during follow-up (ranging from 1.8 to 153 months after surgery) showed good cardiac function with a median ejection fraction (EF) of approximately 57\% (30\%-82\%). The median left ventricular end-diastolic diameter was $51.8 \pm 7.7 \mathrm{~cm}$, and the mean transprosthetic Doppler gradient was $8.5 \pm 4.9 \mathrm{~mm} \mathrm{Hg}$. EF remained stable or even improved up to $30 \%$ in 24 patients 3 months to 1 year after surgery. In 2 patients, a mild reduction of the postoperative $\mathrm{EF}$ was found.

\section{Event-Free Survival}

To evaluate the efficacy of treatment, a composite end point at 30 days of mortality, reoperation for bleeding, and thromboembolic events, and a composite end point of mortality, reoperation for prostheses dysfunction, and recurrence of endocarditis at 5 and 10 years were defined to investigate freedom from treatment failure. Actuarial freedom from events at 30 days was $68.75 \%$, and freedom from events at 5 and 10 years was $56.3 \%$ and $53.1 \%$, respectively.

\section{DISCUSSION}

In this study we aimed to evaluate the comparability of xenograft with homograft root replacement in patients with severe destructive aortic root endocarditis in respect to intraoperative technical complications and long-term outcome.

The present study exclusively included patients with acute IE with extensive root destruction, and only Freestyle root replacements were performed. Additional procedures were performed in more than $50 \%$ of cases. Our results show that there were no instances of technical failure requiring modification of the surgical strategy or reoperations for anastomotic bleeding. The perioperative survival and freedom from events at 30 days, 5 years, and 10 years were acceptable for this severely ill population.

The 20-year experience of a German group studying homograft aortic root replacement in IE shows results similar to our findings with xenografts concerning 30-day survival $(83.8 \%$ vs $81.2 \%)$, recurrence of endocarditis $(5.4 \%$ vs $3.1 \%)$, and valve deterioration $(8.6 \%$ vs $6.3 \%)$ during follow-up. ${ }^{7}$ El-Hamamsy and colleagues ${ }^{8}$ found that the use of the porcine xenograft root is associated with a significantly lower rate of structural valve deterioration and reoperation compared with homograft root replacement. Miceli and colleagues ${ }^{9}$ currently presented low early and mid-term mortality, good hemodynamic performance, low rates of valve-related morbidity, and low recurrent infection, especially in case of PVE, in an 18-patient series with 24 months of follow-up.

The advantages of homografts and stentless xenografts are well known. On the one hand, aortic root homografts have been used for several decades with good long-term results. ${ }^{7,10}$ In comparison with the more obstructive valve prostheses, their lower transvalvular gradients are associated with better left ventricular mass regression. ${ }^{11}$ Homografts show good resistance to infection, and other valve-related complications are rare. ${ }^{12}$ Limited homograft availability determines that they are not used routinely. ${ }^{13}$ Because of low-grade immunologic mechanisms, homografts can undergo late degeneration marked by severe calcification and valve dysfunction. ${ }^{8}$ On the other hand, there is the Freestyle xenograft, which is treated with alpha-oleic acid as an attempt to reduce long-term valve degeneration. ${ }^{8,14}$ Freestyle valves are readily available in different sizes $(19-29 \mathrm{~mm})^{8}$ and storable for even unscheduled use. Although xenograft root replacement may be associated with higher surgical morbidity and mortality because of prolonged myocardial ischemia and more perioperative bleeding complications, ${ }^{15}$ several clinical studies have demonstrated excellent performance.

Recent studies by the Freestyle Valve Study Group have demonstrated the full root implantation of the xenograft to be associated with a superior hemodynamic performance in the long-term follow-up compared with homografts. ${ }^{16,17}$ In a comparison of stentless and stented valves, Funder ${ }^{18}$ stated that these prostheses perform similarly in regard to various clinical parameters and valve-related mortality, but stentless valves have several advantages concerning 
hemodynamic and biomechanical characteristics. In a randomized trial, Lehmann and colleagues ${ }^{19}$ showed a significantly higher long-term survival in stentless compared with stented valve replacement ( $78 \%$ and $66 \%$, respectively) in 223 patients.

Overall excellent hemodynamic results with low gradients at acceptable operative risk can be achieved by fullroot stentless valve replacement. ${ }^{20}$ By comparing our 10 -year survival of $54.2 \%$ with the study of Lehmann and colleagues ${ }^{19}$ with an 8 -year survival of $78 \%$, it must be considered that our patient population underwent operation for excavating aortic root endocarditis only, and in $71.9 \%$ of the cases it was performed as a reoperation with 1 to 4 previous valve surgeries.

In a retrospective study on valve replacement in 77 patients with acute IE, Delay and colleagues ${ }^{21}$ showed that prosthetic valve replacement for NVE resulted in excellent long-term survival without reoperation in both the aortic and mitral positions. For PVE, the 5-year total survival and survival without reoperation were $59 \%$ and $40 \%$, respectively. This outcome of PVE demonstrates a significant difference compared with patients undergoing surgery for NVE. ${ }^{21}$ Further studies also indicate PVE is an independent risk factor for perioperative mortality ${ }^{22,23}$ and limited longterm survival. ${ }^{23,24}$ In contrast to these studies using mainly mechanical or biological valved conduits, our results show excellent xenograft function up to 11 years, with no significant difference between PVE and NVE.

Recurrence of endocarditis in our patients was low $(3.1 \%)$ because of aggressive antibiotic therapy (long duration and number of antibiotics). The result is consistent with that of a French study showing that adequate antimicrobial therapy in IE significantly improves prognosis. ${ }^{25}$ With these findings, it is questionable if the use of a homograft reduces the risk of persistent or recurrent infection compared with a xenograft or if both conduits are likewise effective.

\section{Study Limitations}

This was a retrospective study design with no control group. Although it is the largest published series on the use of the Freestyle in this indication, the number of patients is still small but comparable to published series on the use of homografts.

\section{CONCLUSIONS}

To date, this is the largest reported series of patients with most severe destructive aortic root endocarditis who had repair of the LVOT and aortic root using the Freestyle porcine aortic root. In view of this complex patient population, short- and long-term results make this conduit a reliable choice for treatment of this condition. The xenograft can be used successfully instead of a homograft with comparable or even better long-term durability.

\section{References}

1. Bonow RO, Carabello BA, Chatterjee K, de Leon AC, Faxon DP, Freed MD, et al. 2008 Focused update incorporated into the ACC/AHA 2006 guidelines for the management of patients with valvular heart disease: a report of the American College of Cardiology/American Heart Association Task Force on Practice Guidelines (Writing Committee to Revise the 1998 Guidelines for the Management of Patients With Valvular Heart Disease): endorsed by the Society of Cardiovascular Anesthesiologists, Society for Cardiovascular Angiography and Interventions, and Society of Thoracic Surgeons. Circulation. 2008;118:e523-661.

2. Habib G, Hoen B, Tornos P, Thuny F, Prendergast B, Vilacosta I, et al. Guidelines on the prevention, diagnosis, and treatment of infective endocarditis (new version 2009): the Task Force on the Prevention, Diagnosis, and Treatment of Infective Endocarditis of the European Society of Cardiology (ESC). Endorsed by the European Society of Clinical Microbiology and Infectious Diseases (ESCMID) and the International Society of Chemotherapy (ISC) for Infection and Cancer. Eur Heart J. 2009;30:2369-413.

3. Müller LC, Chevtchik O, Bonatti JO, Müller S, Fille M, Laufer G. Treatment of destructive aortic valve endocarditis with the Freestyle Aortic Root Bioprosthesis. Ann Thorac Surg. 2003;75:453-6.

4. Katsumata T, Vaccari G, Westaby S. Stentless xenograft repair of excavating aortic root sepsis. J Card Surg. 1998;13:440-4.

5. Okada K, Tanaka H, Takahashi H, Morimoto N, Munakata H, Asano M, et al. Aortic root replacement for destructive aortic valve endocarditis with left ventricular-aortic discontinuity. Ann Thorac Surg. 2008;85:940-5.

6. Li JS, Sexton DJ, Mick N, Nettles R, Fowler VG, Ryan T, et al. Proposed modifications to the Duke criteria for the diagnosis of infective endocarditis. Clin Infect Dis. 2000;30:633-8.

7. Musci M, Weng Y, Hübler M, Amiri A, Pasic M, Kosky S, et al. Homograft aortic root replacement in native or prosthetic active infective endocarditis: twentyyear single-center experience. J Thorac Cardiovasc Surg. 2010;139:665-73.

8. El-Hamamsy I, Clark L, Stevens LM, Sarang Z, Melina G, Takkenberg JJ, et al. Late outcomes following freestyle versus homograft aortic root replacement: results from a prospective randomized trial. J Am Coll Cardiol. 2010;55:368-76.

9. Miceli A, Croccia M, Simeoni S, Varone E, Murzi M, Farneti PA, et al. Root replacement with stentless Freestyle bioprostheses for active endocarditis: a single centre experience. Interact Cardiovasc Thorac Surg. 2013;16:27-30.

10. Palka P, Harrocks S, Lange A, Burstow DJ, O'Brien MF. Primary aortic valve replacement with cryopreserved aortic allograft: an echocardiographic follow-up study of 570 patients. Circulation. 2002;105:61-6.

11. Lim E, Ali A, Theodorou P, Sousa I, Ashrafian H, Chamageorgakis T, et al. Longitudinal study of the profile and predictors of left ventricular mass regression after stentless aortic valve replacement. Ann Thorac Surg. 2008;85:2026-9.

12. Lund O, Chandrasekaran V, Grocott-Mason R, Elwidaa H, Mazhar R, Khaghani A, et al. Primary aortic valve replacement with allografts over twenty-five years: valverelated and procedure-related determinants of outcome. J Thorac Cardiovasc Surg. 1999; 117:77-91.

13. Westaby S, Huysmans HA, David TE. Stentless aortic bioprostheses: compelling data from the Second International Symposium. Ann Thorac Surg. 1998;65:235-40.

14. Kon ND, Riley RD, Adair SM, Kitzman DW, Cordell AR. Eight-year results of aortic root replacement with the freestyle stentless porcine aortic root bioprosthesis. Ann Thorac Surg. 2002;73:1817-21.

15. Kon ND, Westaby S, Amarasena N, Pillai R, Cordell AR. Comparison of implantation techniques using freestyle stentless porcine aortic valve. Ann Thorac Surg. 1995;59:857-62.

16. Bach DS, Cartier PC, Kon ND, Johnson KG, Deeb GM, Doty DB, et al. Impact of implant technique following freestyle stentless aortic valve replacement. Ann Thorac Surg. 2002;74:1107-14.

17. Bach DS, Kon ND, Dumesnil JG, Sintek CF, Doty DB. Ten-year outcome after aortic valve replacement with the freestyle stentless bioprosthesis. Ann Thorac Surg. 2005;80:480-7

18. Funder JA. Current status on stentless aortic bioprosthesis: a clinical and experimental perspective. Eur J Cardiothorac Surg. 2012;41:790-9.

19. Lehmann S, Walther T, Kempfert J, Leontjev S, Rastan A, Falk V, et al. Stentless versus conventional xenograft aortic valve replacement: midterm results of a prospectively randomized trial. Ann Thorac Surg. 2007;84:467-72.

20. Dapunt OE, Easo J, Hölzl PP, Murin P, Südkamp M, Horst M, et al. Stentless full roo bioprosthesis in surgery for complex aortic valve-ascending aortic disease: a single center experience of over 300 patients. Eur J Cardiothorac Surg. 2008;33:554-9.

21. Delay D, Pellerin M, Carrier M, Marchand R, Auger P, Perrault LP, et al. Immediate and long-term results of valve replacement for native and prosthetic valve endocarditis. Ann Thorac Surg. 2000;70:1219-23. 
22. Leontyev S, Borger MA, Modi P, Lehmann S, Seeburger J, Doenst T, et al. Surgical management of aortic root abscess: a 13-year experience in 172 patients with $100 \%$ follow-up. J Thorac Cardiovasc Surg. 2012;143: $332-7$.

23. Leontyev S, Borger MA, Modi P, Lehmann S, Seeburger J, Walther T, et al. Redo aortic valve surgery: Influence of prosthetic valve endocarditis on outcomes. J Thorac Cardiovasc Surg. 2011;142:99-105.
24. Garrido-Olivares L, Maganti M, Armstrong S, David TE. Clinical outcomes of aortic root replacement after previous aortic root replacement. J Thorac Cardiovasc Surg. August 20, 2012 [Epub ahead of print].

25. Fayad G, Vincentelli A, Leroy G, Devos P, Amr G, Prat A, et al. Impact of antimicrobial therapy on prognosis of patients requiring valve surgery during active infective endocarditis. J Thorac Cardiovasc Surg. November 7, 2012 [Epub ahead of print].

Access to The Journal of Thoracic and Cardiovascular Surgery Online is reserved for print subscribers!

Full-text access to The Journal of Thoracic and Cardiovascular Surgery Online is available for all print subscribers. To activate your individual online subscription, please visit The Journal of Thoracic and Cardiovascular Surgery Online, point your browser to http://www.mosby.com/itcvs, follow the prompts to activate your online access, and follow the instructions. To activate your account, you will need your subscriber account number, which you can find on your mailing label (note: the number of digits in your subscriber account number varies from 6 to 10). See the example below in which the subscriber account number has been circled:

\section{Sample mailing label}

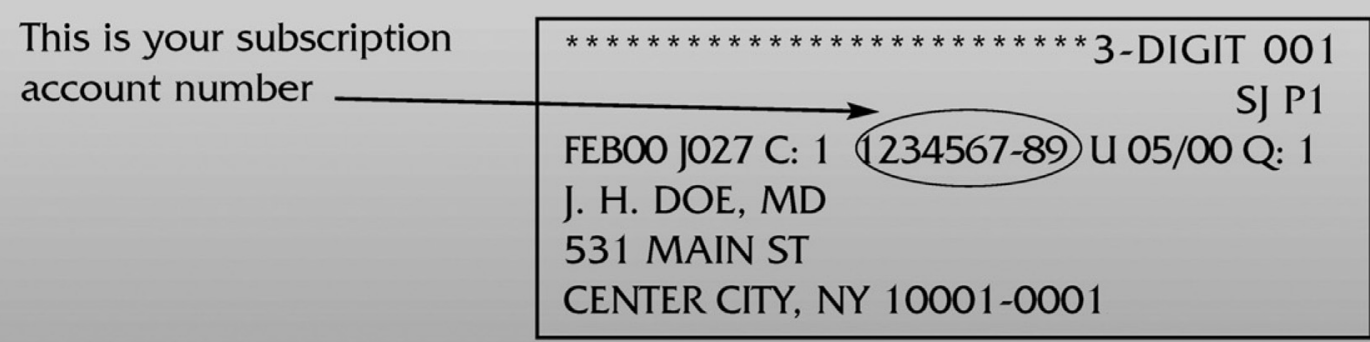

Personal subscriptions to The Journal of Thoracic and Cardiovascular Surgery Online are for individual use only and may not be transferred. Use of The Journal of Thoracic and Cardiovascular Surgery Online is subject to agreement to the terms and conditions as indicated online. 